\section{Hypoglycemia - Old foe with a new face - Masquerading as an acute stroke}

Sir,

A 50-year-old man, known diabetic since 6 months on glibenclamide, presented to us in the emergency department with sudden onset weakness in left upper and lower limb for 3 hours. The patient was afebrile, with a regular pulse rate of $78 / \mathrm{min}$ and blood pressure of $130 / 70 \mathrm{mmHg}$. He was conscious but confused. Power in left upper limb was grade 1 and lower limb grade 3. Cranial nerves were normal on examination. The National Institute of Health Stroke Score (NIHSS) was 6 on admission. Rest of the physical examination was normal. The patient was immediately shifted to magnetic resonance imaging (MRI) for a scan. The emergency laboratory reports showed a random blood sugar of $40 \mathrm{mg} / \mathrm{dl}$. Liver and renal function tests, lipid profile, electrolytes (calcium, sodium, potassium, phosphate), and hemogram were normal. On questioning, it was found that the patient had taken an extra tablet of glibenclamide. $100 \mathrm{ml}$ of $25 \%$ dextrose solution was given. Repeat blood sugar was $77 \mathrm{mg} / \mathrm{dl}$. The confusion improved, power normalized without any residual neurodeficit and repeat NIHSS was 0 . Surprisingly, patient's MRI-diffusion weighted imaging (DWI) and activated diffusion coefficient (ADC) was suggestive of hyperacute ischemia in posterior limb of the right internal capsule [Figures 1 and 2]. However, as it did not appear hyperintense on fluid-attenuated inversion recovery sequence, hyperacute ischemia was suspected. The magnetic resonance angiogram was normal.

Although the patient recovered fully with no residual deficit, treatment with antiplatelets and statin was started. An endocrinology consultation was made. The patient was counseled about hypoglycemic symptoms and dosage of oral hypoglycemic drugs were adjusted. On follow-up at 6 weeks, the patient was completely asymptomatic with no neurodeficit.

Hypoglycemia in diabetic patients can occur because of dietetic errors, alcohol abuse, excessive physical effort, or error in the dose of oral hypoglycemic agent (OHA), especially in elderly patients. Nonischemic causes account for around $13 \%$ of cases ${ }^{[1]}$ Hemiparesis is a rare sign of hypoglycemia as usually ischemia, and hemorrhagic events are considered the most common events for hemiparesis in an elderly in the clinical setting of risk factors. Hemiparesis secondary to hypoglycemia has been reported as early as $1928 .^{[2]}$ Patients experience transient hemiparesis with retained alertness during periods of hypoglycemia, which resolves with correction of hypoglycemia. Surprisingly, the manifestations of hypoglycemia (hunger, diaphoresis, generalized weakness, dizziness) are usually absent during attacks of hypoglycemia-induced hemiparesis $(\mathrm{HH}){ }^{[3]}$

In neonates, hypoglycemia is very common, with occipital lobes being involved more than parietal lobe. This is due to a regional deficit in expression and function of the glucose membrane transporter proteins. However, in adults, the regions involved are basal ganglia, hippocampus, insula, frontal regions, and corpus callosum. Pathogenesis of neuronal damage during hypoglycemic episodes involves cytotoxic edema, shrinkage of the extracellular space (ECS) and failure of ionic pumps of the cell membrane after energy depletion..$^{[2]}$ Hypoglycemic brain injury is widely studied in patients with hypoglycemic coma showing the involvement of cerebral cortex, basal ganglia, and hippocampus. In patients presenting with hemiparesis, the internal capsule and splenium were often affected, suggesting that these areas are more vulnerable. ${ }^{[4]}$ Local neurodeficit may also occur after seizures. A 2-3-fold increase in glucose utilization

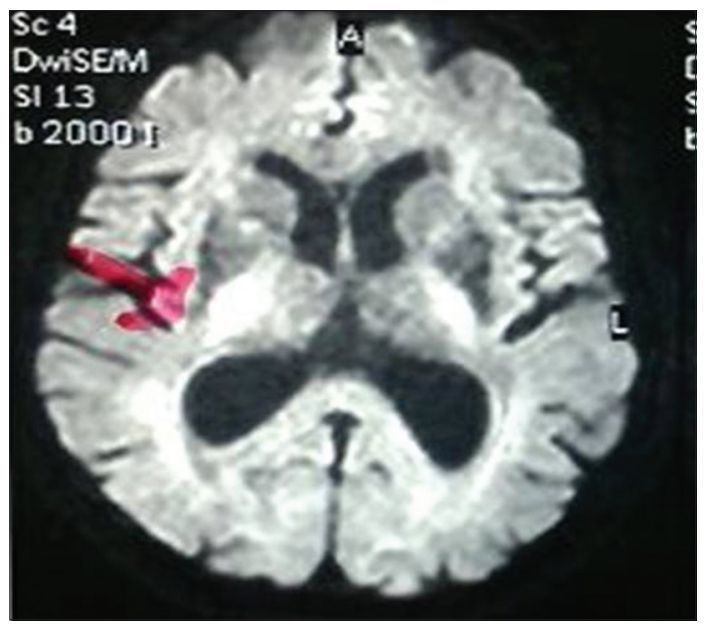

Figure I: Magnetic resonance imaging brain diffusion weighted imaging showing restricted diffusion in posterior limb of the right internal capsule 


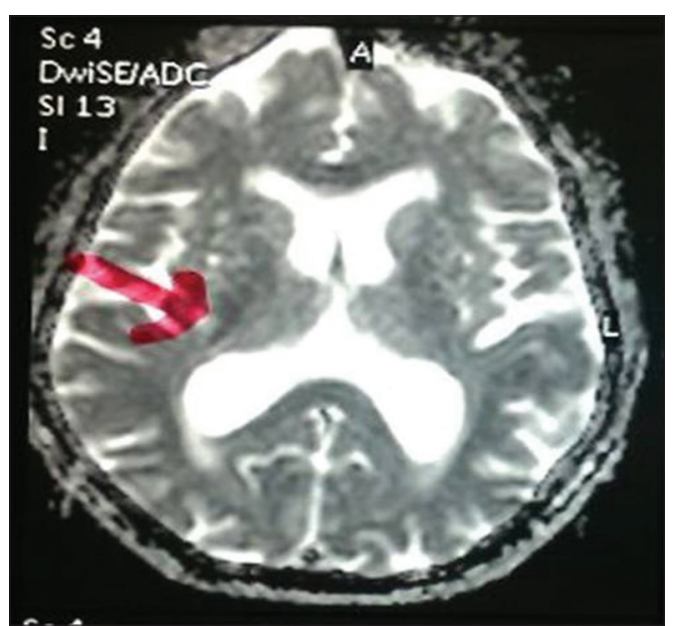

Figure 2: Magnetic resonance imaging brain activated diffusion coefficient showing corresponding hypointensity in the same area as Figure I (shown by arrow)

during seizure discharges and suggested paralysis that follows (Todd's postepileptic paralysis) might be a result of neuronal depletion of glucose and an increase of lactic acid. ${ }^{[5]}$ In our case, there was no history suggestive of seizure. Based on 29 well-documented cases, in a study conducted at Harlem Hospital, the clinical spectrum of $\mathrm{HH}$ not only occurs in diabetic patients receiving insulin $(72 \%)$ or OHA $(14 \%)$, but also in nondiabetic patients with other causes of hypoglycemia (14\%). The mean serum glucose during $\mathrm{HH}$ is $35 \mathrm{mg} \%$. On average, most patients experience 3.5 attacks before $\mathrm{HH}$ is recognized and treated. Attacks of hemiparesis alternate, involving both the left and right sides, in $29 \%$ of cases. Curiously, right hemiparesis is far more common than left (72\% vs. $28 \%)$ and is often accompanied by aphasia. Paralysis may be either flaccid or, less often, spastic. The duration of $\mathrm{HH}$ is, in general, somewhat longer than the usual duration of transient ischemic attack due to cerebrovascular disease. ${ }^{[6]}$

MRI-DWI and ADC mapping, are the most useful tools in diagnosing the early ischemic injury. Ischemia results in metabolic energy failure with disruption of water and electrolyte homeostasis consecutively leading to impairment of cell membrane potential. This provokes an osmotically driven flow of water intracellularly and a consecutive reduction of the ECS which contribute to the changes of $\mathrm{ADC}$ in ischemia. Complete/partial recovery of ADC reductions could be demonstrated in transient ischemia, suggesting that early ADC decreases do not necessarily represent irreversible tissue damage. ADC reductions are not unique to ischemia and occur in other conditions, such as seizures, spreading depression, and excitotoxic cerebral injuries, as well as hypoglycemia. However, the pathophysiology of these conditions for ADC changes are heterogeneous. ${ }^{[4]}$
In patients with diabetes mellitus, receiving either insulin or OHA, the presence of focal or general neurologic symptoms requires the consideration of hypoglycemia as a possible cause. Especially in diabetic patients with autonomic neuropathy, blunting of the catecholamine response to hypoglycemia may result in isolated neuroglycopenic symptoms.

\section{Financial support and sponsorship \\ Nil.}

\section{Conflicts of interest}

There are no conflicts of interest.

\section{Virti D. Shah, Sumeet Prakash Mirgh ${ }^{1}$, Rishit K. Harbada ${ }^{2}$, Nirmal Surya ${ }^{3}$} Department of Neurology, Sir Ganga Ram Hospital, 'Department of Hematology, AlIMS, New Delhi, Departments of ${ }^{2}$ Medicine and ${ }^{3}$ Neurology, Bombay Hospital Institute of Medical Sciences, Mumbai, Maharashtra, India

Correspondence: Dr. Sumeet Prakash Mirgh, No 401, Jai Gurudev C.H.S., Plot No 6, Sector No 1, Sanpada, Navi Mumbai - 400 705, Maharashtra, India.
E-mail: drsumeetmirgh @ gmail.com

\section{References}

1. Sylaja PN, Coutts SB, Krol A, Hill MD, Demchuk AM; VISION Study Group. When to expect negative diffusion-weighted images in stroke and transient ischemic attack. Stroke 2008;39:1898-900.

2. Yoshino T, Meguro S, Soeda Y, Itoh A, Kawai T, Itoh H. A case of hypoglycemic hemiparesis and literature review. Ups J Med Sci $2012 ; 117: 347-51$.

3. Wallis WE, Donaldson I, Scott RS, Wilson J. Hypoglycemia masquerading as cerebrovascular disease (hypoglycemic hemiplegia). Ann Neurol 1985;18:510-2.

4. Chan R, Erbay S, Oljeski S, Thaler D, Bhadelia R. Case report: Hypoglycemia and diffusion-weighted imaging. J Comput Assist Tomogr 2003;27:420-3.

5. Plum F, Howse DC, Duffy TE. Metabolic effects of seizures. Assoc Res Nerv Ment Dis 1974;53:141-57.

6. Foster JW, Hart RG, Oljeski S, Thaler D, Bhadelia R. Hypoglycemic hemiplegia: Two cases and a clinical review. Stroke 1987;18:944-6.

This is an open access article distributed under the terms of the Creative Commons Attribution-NonCommercial-ShareAlike 3.0 License, which allows others to remix, tweak, and build upon the work non-commercially, as long as the author is credited and the new creations are licensed under the identical terms.

\begin{tabular}{|l|l|}
\hline \multicolumn{2}{|c|}{ Access this article online } \\
\hline Quick Response Code: & Website: \\
& www.ijccm.org \\
\cline { 2 - 3 } & DOI: $10.4103 / 0972-5229.194010$ \\
\hline
\end{tabular}

How to cite this article: Shah VD, Mirgh SP, Harbada RK, Surya N. Hypoglycemia - Old foe with a new face - Masquerading as an acute stroke. Indian J Crit Care Med 2016;20:684-5. 